\title{
Less than $2 \%$ of the Low- and Intermediate-Risk Differentiated Thyroid Cancers Show Distant Metastases at Post-Ablation Whole-Body Scan
}

\author{
Laura Agate $^{a}$ Francesca Bianchi $^{b}$ Federica Brozzi ${ }^{b}$ Pierina Santini ${ }^{b}$ \\ Eleonora Molinaro $^{a}$ Valeria Botticia ${ }^{a}$ David Viola ${ }^{a}$ Loredana Lorusso ${ }^{a}$ \\ Paolo Vitti $^{\mathrm{a}}$ Rossella Elisei ${ }^{\mathrm{a}}$

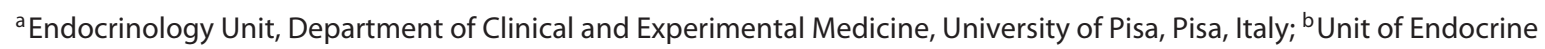 \\ and Oncological Nuclear Medicine Therapy, Diagnostic and Imaging Department, University of Pisa, Pisa, Italy
}

\section{Keywords}

Thyroid remnant ablation · Neck ultrasound $\cdot$ Thyroid

cancer · Distant metastases . Whole-body scan

\begin{abstract}
Background: Recently, there has been a trend to reduce the use of radioiodine remnant ablation (RRA) in patients with low-risk (LR) and intermediate-risk (IR) differentiated thyroid cancer (DTC). Objectives: The aim of this paper was to evaluate the diagnostic role of whole-body scan (ptWBS) performed after RRA in LR and IR DTC patients. Methods: We analyzed 545 DTC patients treated with total thyroidectomy and RRA in hypothyroidism followed by a ptWBS. Neck ultrasound (US) and serum thyroglobulin measurement were performed. According to the American Thyroid Association guidelines, patients were classified as LR $(n=345)$ and IR ( $n=200)$. Results: In addition to the thyroid remnant, the ptWBS showed the presence of further areas of ${ }^{131}$ I uptake in $16 / 545$ (2.9\%) cases. ptWBS showed laterocervical lymph node metastases in $11 / 16$ patients (10/11 were also detected by US), mediastinal uptake in 1/16, lung metastases in $3 / 16$, and bone metastases in 1/16. Only 6/545 (1.1\%) metastases were detected by ptWBS alone. After 7.8 years, $8 / 16$ patients
\end{abstract}

were free of disease, and 8 had persistent disease: 4 "biochemical" and 4 "structural." Remission was achieved in 3 cases after one single ${ }^{131}$ I course, in 1 case after surgery, and in the last 4 cases after several ${ }^{131}$ I courses. Conclusions: The ptWBS diagnostic role was clinically relevant for the therapeutic strategies of our patients only in $1.1 \%$ of the cases. The cost-effectiveness of performing RRA and ptWBS in all LR and IR patients to find $1-2 \%$ of the cases with distant metastases remains controversial.

(c) 2018 European Thyroid Association Published by S. Karger AG, Basel

\section{Introduction}

Until a few years ago, the standard therapy of welldifferentiated thyroid cancer (DTC) consisted of total or near total thyroidectomy followed by radioiodine (RAI) remnant ablation (RRA) and TSH suppressive levothyroxine (LT4) therapy [1]. The rationale for RRA included, and still includes, three major purposes: (a) the therapeutic purpose related to the irradiation of any neoplastic microfocus that can be present in about $50 \%$ of the thyroid remnant, especially in the case of papillary thyroid carci-

\section{KARGER}

E-Mail karger@karger.com

www.karger.com/etj (c) 2018 European Thyroid Association

Published by S. Karger AG, Basel
Rossella Elisei, MD

Endocrinology Unit, Department of Clinical and Experimental Medicine University of Pisa

Via Paradisa 2, IT-56124 Pisa (Italy)

E-Mail rossella.elisei@med.unipi.it 
noma (PTC); (b) the diagnostic purpose due to the possibility to perform a post-RRA whole-body scan (ptWBS) to identify additional sites of disease not identified before the RRA [2-4]; and (c) the facilitator purpose because the ablation of residual normal thyroid tissue, commonly producing thyroglobulin ( $\mathrm{Tg}$ ), makes serum $\mathrm{Tg}$ measurements much more sensitive and predictive of recurrent or persistent disease.

In recent years, there has been a trend to reduce the use of RRA to limit the potential secondary side effects, particularly in patients with low-risk (LR) and intermediaterisk (IR) DTC, even if these effects are negligible for RAI activities $<100 \mathrm{mCi}$ [5-7]. Nowadays, there is a general agreement that LR DTC should not be submitted to RRA $[8,9]$ since a significant increase in disease-free survival or decrease in mortality has been demonstrated in these patients [10-14]. On the opposite, the use of RRA, usually with high activities of ${ }^{131} \mathrm{I}$, is recommended for the management of high-risk patients in whom this procedure has shown a positive impact on recurrence and survival $[15,16]$. A selective use of RRA is proposed for the IR patients taking into account the level of risk of recurrence as well as the values of postoperative serum $\mathrm{Tg}$ and neck ultrasound (US) $[17,18]$.

Despite the evidence of a limited therapeutic role of RRA in LR and IR DTC, to our knowledge, no studies on the benefits of the diagnostic role of ptWBS have been reported so far. Recently, we have published a study showing that only $1.3 \%$ of the LR and IR DTC patients showed a positive ptWBS when treated with $30 \mathrm{mCi}$ and after recombinant human TSH (rhTSH) stimulation [18]. One criticism to this study could be that the sensitivity of the ptWBS after rhTSH might be much lower than after the stimulus of an elevated endogenous TSH obtained after LT4 withdrawal. The main objective of the present study was to evaluate the diagnostic role of ptWBS performed after RRA in LR and IR DTC patients prepared with LT4 withdrawal and, as a secondary objective, to verify how much the derived information could change the therapeutic strategies for these patients.

\section{Patients and Methods}

Study Groups

We analyzed the data of 545 consecutive patients ( 410 females [75.2\%], 135 males [24.8\%]) with LR $(n=345)$ or IR $(n=200)$ DTC treated with total thyroidectomy and referred to the Department of Endocrinology of Pisa for RRA during the year 2006. According to TNM and the American Thyroid Association guidelines for thyroid cancer management $[8,19]$, LR consisted of T1-2mN0/xM0/x patients, and IR included T1-3N1Mx and T3N0Mx patients.
Table 1. Results of ptWBS in LR and IR DTC patients

\begin{tabular}{lcc}
\hline & $\begin{array}{l}\text { LR } \\
(n=345)\end{array}$ & $\begin{array}{l}\text { IR } \\
(n=200)\end{array}$ \\
\hline TR & 340 & 189 \\
TR+lymph node mets & 3 & 8 \\
TR+mediastinal mets & 0 & 1 \\
TR+lung mets & 1 & 2 \\
TR+bone mets & 1 & 0 \\
\hline
\end{tabular}

TR, thyroid remnant; mets, metastasis.

The mean age at diagnosis was $43.3 \pm 13.4$ years, with a median of 43.5 years. A total of 527 (96.7\%) patients had PTC, and $18(3.3 \%)$ had follicular thyroid cancer. Because the use of recombinant TSH was not yet part of clinical practice, all patients were treated for hypothyroidism obtained with LT4 withdrawal. According to our standard of care for LR and IR DTC patients, everybody was treated with a "low" activity of $30 \mathrm{mCi}$ of ${ }^{131} \mathrm{I}(1,110 \mathrm{MBq})$ followed by ptWBS. Neck US, serum Tg, thyroid hormone measurements, and urinary iodine secretion were performed in all patients.

\section{Biochemical Tests}

All biochemical tests were conducted in the same laboratory of the Department of Endocrinology in Pisa. Serum Tg was measured using an immunometric assay (Immulite 2000 Tg; Diagnostic Products Corporation, Los Angeles, CA, USA) with a functional sensitivity of $0.9 \mathrm{ng} / \mathrm{mL}$. TSH measurement was performed using an ultrasensitive immunochemiluminescent assay method (Immulite 2000 TSH; Diagnostic Products Corporation).

To avoid misinterpretation of Tg measurements, all patients were screened for circulating anti-Tg antibodies, using an immunofluorimetric method (AIA-Pack TgAb; Tosoh Corporation, Tokyo, Japan; normal range: $0-30 \mathrm{U} / \mathrm{mL}$ ). Urinary iodine content was measured with the UV irradiation method employing an automated autoanalyzer (Bran-Luebbe S.P.A., Gallarate, Italy) [20].

\section{Neck US}

US was performed using a color doppler apparatus (AU 590 Asynchronous; Esaote Biomediva, Florence, Italy) in all patients before RRA. The thyroid bed and central, laterocervical and supraclavear node compartments were evaluated for the presence of suspicious disease recurrence or persistence. In suspicious lymph node metastases, a fine-needle aspiration biopsy with Tg measurement in the needle washout was performed.

\section{${ }^{131} I-p t W B S$}

The ptWBS was performed using a dual-head large field-ofview gamma camera (Philips Axis; Picker International, Inc., Highlands Heights, OH, USA) with a 6/8-inch-thick crystal and equipped with high-energy high-resolution collimators. The ptWBS was performed 5-10 days after the ${ }^{131}$ I treatment on the basis of a $50 \mu \mathrm{Ci}$ thyroid bed RAI 24-h uptake (New Atomlab 950; Biodex Medical System, Urbino, Italy).

In all patients, a low activity of ${ }^{131} \mathrm{I}(30 \mathrm{mCi})$ was administered providing that the TSH value was $>30 \mu \mathrm{U} / \mathrm{mL}$ and urinary iodine content $<300 \mathrm{mcg} / \mathrm{L}$. 
Table 2. Clinical and anatomopathological features of $6 / 545$ (1.1\%) patients with metastases documented only by ptWBS

\begin{tabular}{|c|c|c|c|c|c|c|c|c|}
\hline Patient & $\begin{array}{l}\text { Site of } \\
\text { metastases }\end{array}$ & $\begin{array}{l}\text { Age at } \\
\text { diagnosis, } \\
\text { years }\end{array}$ & Sex & $\begin{array}{l}\text { Histology } \\
\text { (variant) }\end{array}$ & TNM & $\begin{array}{l}\text { Size, } \\
\mathrm{cm}\end{array}$ & $\begin{array}{l}\text { Serum } \\
\mathrm{Tg}, \\
\mathrm{ng} / \mathrm{mL}\end{array}$ & Outcome \\
\hline 1 & Bone & 40 & $\mathrm{~F}$ & PTC (follicular) & $\mathrm{T} 1 \mathrm{NxMx}$ & 1 & 97 & Structural disease \\
\hline 2 & Mediastinal lymph node & 68 & M & PTC (follicular) & T3N1aMx & Unkown & 0.5 & Structural disease \\
\hline 3 & Laterocervical lymph node & 28 & $\mathrm{M}$ & PTC (follicular) & $\mathrm{T} 2 \mathrm{NxMx}$ & 2.3 & 2.4 & Remission \\
\hline 4 & Lung & 34 & $\mathrm{~F}$ & PTC (classic) & T3N1aMx & 0.5 & 733 & $\begin{array}{l}\text { Biochemical } \\
\text { disease }\end{array}$ \\
\hline 5 & Lung & 23 & $\mathrm{~F}$ & PTC (classic) & T3N1aMx & 4.3 & 4 & Remission \\
\hline 6 & Lung & 37 & $\mathrm{~F}$ & PTC (follicular) & $\mathrm{T} 2 \mathrm{NxMx}$ & 4.5 & 167 & Remission \\
\hline
\end{tabular}

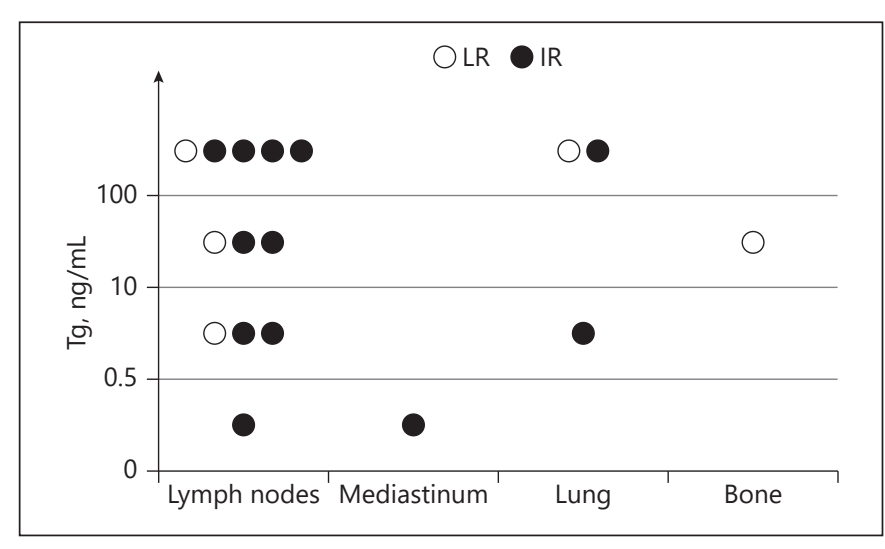

Fig. 1. Serum Tg values in patients with ${ }^{131} \mathrm{I}$ uptake outside the thyroid bed detected by ptWBS. No significant correlation was found between the serum Tg values and metastatic lesions detected and the level of risk.

\section{Statistical Analysis}

The statistical analysis of data was performed by Mann-Whitney $U$ and $\chi^{2}$ tests using a computer program Statview 4.5 software (Abacus Concepts Inc., Berkley, CA, USA). The results were considered statistically significant if $p<0.05$.

\section{Results}

\section{ptWBS Findings}

The thyroid remnant was documented in all patients at ptWBS. In addition to this finding, the ptWBS showed the presence of further areas of ${ }^{131}$ I uptake in $16 / 545$ patients $(2.9 \% ; \mathrm{F}=11$ and $\mathrm{M}=5)$. According to the level of risk of recurrence, 5 patients were classified as LR $(2.1 \%)$ and 11 as IR $(5.5 \%$; $p=\mathrm{ns})$.

The histology was PTC in all 16 cases, 6 of whom showed a more aggressive variant ( 2 tall cells, 1 follicular with solid areas, 1 follicular with tall cells, 1 follicular with oxyphil areas, and 1 poorly differentiated papillary). As shown in Table 1, ptWBS showed the presence of laterocervical lymph node metastases in $11 / 16$ patients ( $3 \mathrm{LR}, 8$ IR), mediastinal uptake in $1 / 16$ (IR, lung metastases in 3/16 [1 LR, 2 IR], either nummular or diffuse), and bone metastases in 1/16 (LR). Moreover, 10 out of 11 laterocervical lymph node metastases were also detected by neck US and confirmed by fine-needle aspiration cytology. Therefore, only 6/545 (1.1\%) cases (3 LR [0.87\%] and 3 IR [1.5\%]) showed metastases ( 1 cervical and 1 mediastinal lymph node, 3 lung and 1 bone metastatic lesions) documented by ptWBS only. None of the patients with lung and bone metastases had aggressive variants in their histological findings. The clinical and anatomopathological features of the above patients are reported in Table 2.

\section{Correlation between Serum $\mathrm{Tg}$ and Metastases}

When considering the subgroup with metastases, the mean value of serum Tg off LT4 at the time of ablation, which can be considered the postsurgical control, was $115.4 \pm 123.2 \mathrm{ng} / \mathrm{mL}$ in the LR group (range: $2.4-300 \mathrm{ng} /$ $\mathrm{mL}$; median: 97 ) and $200.7 \pm 250.9 \mathrm{ng} / \mathrm{mL}$ in the IR group (range: $0-733 \mathrm{ng} / \mathrm{mL}$; median: 60 ) with no statistically significant differences between the two groups. At variance, the mean and median values of serum Tg in a subgroup of patients with only thyroid remnant (i.e., 230/529) were 11.1 and $7 \mathrm{ng} / \mathrm{mL}$, respectively. No significant correlation was found between serum $\mathrm{Tg}$ values and metastatic lesions detected by ptWBS. Three out of 4 cases with distant metastases had a serum $\mathrm{Tg}$ value $>10 \mathrm{ng} / \mathrm{mL}$ (Fig. 1).

\section{Outcome}

At the end of follow-up (mean 7.8 years), 8/16 patients with a positive ptWBS were free of disease (3 LR, 5 IR), 


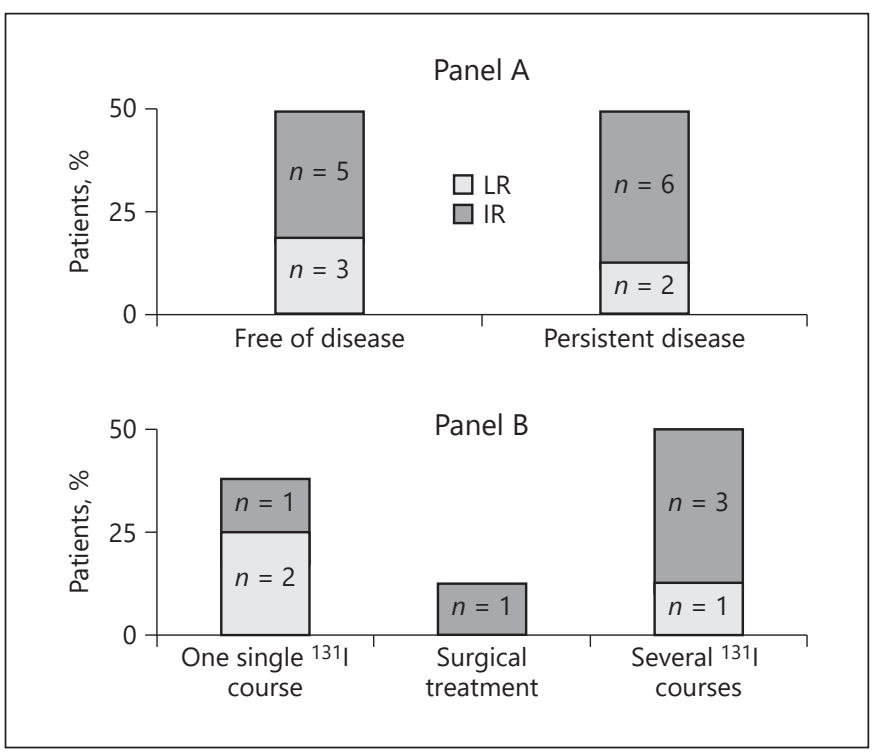

Fig. 2. Panel A: outcomes of LR and IR patients with extrathyroidal positive ptWBS at the end of follow-up (median: 7.8 years). Eight out of 16 patients were free of disease ( 3 LR, 5 IR), whereas the other 8 had persistent disease (2 LR, 6 IR), with no difference between LR and IR. Panel B: treatments used to achieve remission in LR and IR patients with extrathyroidal positive ptWBS. Remission was achieved in 3 cases after one ${ }^{131}$ I course ( 2 LR, 1 IR), in 1 case after surgical treatment (IR), and in the last 4 cases after several ${ }^{131} \mathrm{I}$ courses (1 LR, 3 IR). No major differences in the therapeutic strategies employed during follow-up were observed.

whereas the other 8 had persistent disease (2 LR, 6 IR), without any difference of outcome according to the original level of risk (Fig. 2, panel A). Of the 8 cases with persistent disease, 4 had "biochemical" disease and 4 had "structural" disease (2 lymph nodes, 1 mediastinum, and 1 bone). The 8 cases that became free of disease during follow-up obtained this result after one single course of ${ }^{131}$ I in 3 cases (2 LR, 1 IR), after surgical treatment in 1 case (IR), and after several ${ }^{131}$ I courses in the last 4 cases (1 LR, 3 IR). The outcome of these patients was independent from the original level of risk (Fig. 2, panel B).

\section{Discussion}

The American Joint Committee on the Cancer TNM Staging System is currently the most accepted staging system for DTC patients to predict the risk of death [8]. However, the AJCC/TNM risk of mortality staging system does not adequately predict the risk of recurrences. Thus, the 2009 ATA Initial Stratification System pro- posed 3 disease recurrence risk categories, low, intermediate and high, that were validated in several recent retrospective studies [11, 12, 19]. In addition, this method was in accordance with the evidence that the death rates in both LR and IR DTC patients are extremely low [13, 14].

Today, clinicians are faced with a growing number of patients with LR and IR DTC, and there is a need for safe, efficient, and cost-effective management strategies, avoiding overtreatment, and preserving the quality of life. In particular, despite the fact that RRA has traditionally been one of the cornerstones of DTC treatment, the decision to use RRA in LR and IR patients is more and more controversial [21].

Currently, there is no evidence of a clinical benefit in terms of reducing recurrences and prolonging survival secondary to RRA in LR and IR DTC patients. However, no studies have been published on the diagnostic role of RRA, which is related to the higher sensitivity of ptWBS in finding metastases not detected by other imaging techniques. We have recently published a series of LR and IR DTC in which the ptWBS detected only 1.3\% (7/505) of the cases with distant metastases that, without the administration of RRA, likely would have been diagnosed much later. These patients were prepared with rhTSH for RRA, and some concerns about a lower sensitivity of the ptWBS because of this type of preparation have arisen.

In the present study, we analyzed the ptWBS results in 345 LR patients and 200 IR patients treated with 1,110 $\mathrm{MBq}$ of ${ }^{131}$ I for RRA after LT4 withdrawal. The ptWBS played a diagnostic role in only $2.9 \%$ of the patients after RRA because it identified local and/or distant metastases, with no difference between LR and IR cases. In addition to the thyroid remnant that was present in all cases, ptWBS showed the presence of laterocervical lymph node metastases in 11/16 cases, mediastinal lymph node metastases in 1/16 cases, lung metastases in $3 / 16$ cases, and bone metastases in $1 / 16$ cases.

As far as lymph node metastases are concerned, 10/11 were also detected by neck US and confirmed by cytology examination. This evidence limited the diagnostic role of ptWBS to $6 / 545$ (1.1\%) cases, which is superimposed to the $1.3 \%$ that we found in the series of LR and IR patients prepared with rhTSH for RRA [18]. Moreover, this finding confirmed the excellent accuracy of neck US for the study of neck metastatic lesions [22]. Of the remaining 6 cases, 1 had laterocervical lymph node metastasis, 1 had mediastinal lymph node metastasis, 3 had lung metastases, and 1 had bone lesion metastasis that could not be found without the use of ptWBS. It is of interest that 
among these 6 cases, 3 were LR patients, 3 were IR patients, and only 1 showed a more aggressive histological variant. This finding greatly reduces the prognostic significance of the level of risk in predicting the presence of metastatic lesions out of the neck.

Despite the absence of a specific correlation between serum $\mathrm{Tg}$ levels and distant metastases, $2 / 3$ cases with lung metastases ( $1 \mathrm{LR}$ and $1 \mathrm{IR}$ cases) had a post-thyroidectomy serum $\mathrm{Tg}>100 \mathrm{ng} / \mathrm{mL}$ that could create at least the doubt of metastatic disease and bring the clinician to perform other diagnostic imaging tests or to proceed with RRA and ptWBS without uncertainty. However, the postthyroidectomy serum $\mathrm{Tg}$ was not suspicious in the other 4 cases ( 1 bone and the other lung metastasis, 1 mediastinal, and 1 laterocervical lymph node metastasis). It is likely that these cases would have had a different outcome if the ptWBS had not been performed. In fact, most of them were finally cured with the appropriate therapeutic strategies [23], and we cannot determine if this would have happened if the diagnosis of the metastatic lesions had come later.

In conclusion, we found that the diagnostic role of ptWBS was significant and relevant for the therapeutic strategies only in $1.1 \%$ of our LR and IR patients prepared with LT4 withdrawal, similarly to the series of LR and IR patients prepared with rhTSH (1.3\%). This finding confirms that the sensitivity of the ptWBS was not affected by the type of preparation (i.e., LT4 withdrawal vs. $\operatorname{rhTSH}$ ).
We found no differences according to the initial level of risk since the metastatic cases represented $0.87 \%$ of the LR and $1.5 \%$ of the IR patients. As previously stated, we do not know what would have happened to these patients if RRA had not been performed. However, $30 \%$ of them would not have been overlooked. In fact, they would have been treated with RRA anyway because of the elevated serum Tg levels at the postoperative control, confirming the relevance of serum $\mathrm{Tg}$ at this time of the follow-up of DTC patients [18]. Similarly, the 10 cases with metastatic lymph node at neck US would have been treated anyway because of this finding. The cost-effectiveness of performing RRA and ptWBS in all LR and IR patients, either after LT4 withdrawal or rhTSH, to find 1-2\% of the cases with distant metastases remains to be established but is rather unlikely.

\section{Statement of Ethics}

All patients signed an informed consent to the use of their clinical, biochemical, and pathological data for the purpose of this study that was approved by the institutional review board.

\section{Disclosure Statement}

We declare that there is no conflict of interest that could be perceived as prejudicing the impartiality of the research reported.

\section{References}

1 Mazzaferri EL, Jhiang SM. Long-term impact of initial surgical and medical therapy on papillary and follicular thyroid cancer. Am J Med. 1994 Nov;97(5):418-28.

2 Pacini F, Molinaro E, Lippi F, Castagna MG, Agate L, Ceccarelli C, et al. Prediction of disease status by recombinant human TSH-stimulated serum $\mathrm{Tg}$ in the postsurgical follow-up of differentiated thyroid carcinoma. J Clin Endocrinol Metab. 2001 Dec;86(12):5686-90.

3 Pacini F, Schlumberger M, Dralle H, Elisei R, Smit JW, Wiersinga W; European Thyroid Cancer Taskforce. European consensus for the management of patients with differentiated thyroid carcinoma of the follicular epithelium. Eur J Endocrinol. 2006 Jun;154(6): 787-803.

4 Sawka AM, Brierley JD, Tsang RW, Thabane L, Rotstein L, Gafni A, et al. An updated systematic review and commentary examining the effectiveness of radioactive iodine remnant ablation in well-differentiated thyroid cancer. Endocrinol Metab Clin North Am. 2008 Jun;37(2):457-80.
5 Cherk MH, Kalff V, Yap KS, Bailey M, Topliss D, Kelly MJ. Incidence of radiation thyroiditis and thyroid remnant ablation success rates following $1110 \mathrm{MBq}(30 \mathrm{mCi})$ and $3700 \mathrm{MBq}$ $(100 \mathrm{mCi})$ post-surgical 131I ablation therapy for differentiated thyroid carcinoma. Clin Endocrinol (Oxf). 2008 Dec;69(6):957-62.

6 Canale D, Ceccarelli C, Caglieresi C, Moscatelli A, Gavioli S, Santini P, et al. Effects of radioiodine treatment for differentiated thyroid cancer on testis function. Clin Endocrinol (Oxf). 2015 Feb;82(2):295-9.

7 Clement SC, Peeters RP, Ronckers CM, Links TP, van den Heuvel-Eibrink MM, Nieveen van Dijkum EJ, et al. Intermediate and longterm adverse effects of radioiodine therapy for differentiated thyroid carcinoma-a systematic review. Cancer Treat Rev. 2015 Dec; 41(10):925-34.

8 Piemonte M: [TNM - classification of malignant tumors (VI edition - 2002). Innovations in the classification of head and neck neoplasms]. Acta Otorhinolaryngol Ital. 2003 Apr;23(2):132-35.
9 Kim HJ, Kim NK, Choi JH, Kim SW, Jin SM, Suh S, et al. Radioactive iodine ablation does not prevent recurrences in patients with papillary thyroid microcarcinoma. Clin Endocrinol (Oxf). 2013 Apr;78(4):614-20.

10 Lamartina L, Durante C, Filetti S, Cooper DS Low-risk differentiated thyroid cancer and radioiodine remnant ablation: a systematic review of the literature. J Clin Endocrinol Metab. 2015 May;100(5):1748-61.

11 Tuttle RM, Tala H, Shah J, Leboeuf R, Ghossein R, Gonen M, et al. Estimating risk of recurrence in differentiated thyroid cancer after total thyroidectomy and radioactive iodine remnant ablation: using response to therapy variables to modify the initial risk estimates predicted by the new American Thyroid Association staging system. Thyroid. 2010 Dec; 20(12):1341-9.

12 Vaisman F, Momesso D, Bulzico DA, Pessoa $\mathrm{CH}$, Dias F, Corbo R, et al. Spontaneous remission in thyroid cancer patients after biochemical incomplete response to initial therapy. Clin Endocrinol (Oxf). 2012 Jul;77(1):132-8. 
13 Durante C, Montesano T, Torlontano M, Attard M, Monzani F, Tumino S, et al.; PTC Study Group. Papillary thyroid cancer: time course of recurrences during postsurgery surveillance. J Clin Endocrinol Metab. 2013 Feb; 98(2):636-42.

14 Padovani RP, Robenshtok E, Brokhin M, Tuttle RM. Even without additional therapy, serum thyroglobulin concentrations often decline for years after total thyroidectomy and radioactive remnant ablation in patients with differentiated thyroid cancer. Thyroid. 2012 Aug;22(8):778-83.

15 Jonklaas J, Sarlis NJ, Litofsky D, Ain KB, Bigos ST, Brierley JD, et al. Outcomes of patients with differentiated thyroid carcinoma following initial therapy. Thyroid. 2006 Dec; 16(12):1229-42.

16 Podnos YD, Smith DD, Wagman LD, Ellenhorn JD. Survival in patients with papillary thyroid cancer is not affected by the use of radioactive isotope. J Surg Oncol. 2007 Jul; 96(1):3-7.
17 Haugen BR, Alexander EK, Bible KC, Doherty GM, Mandel SJ, Nikiforov YE, et al. 2015 American Thyroid Association Management Guidelines for Adult Patients with Thyroid Nodules and Differentiated Thyroid Cancer: The American Thyroid Association Guidelines Task Force on Thyroid Nodules and Differentiated Thyroid Cancer. Thyroid. 2016 Jan;26(1):1-133.

18 Matrone A, Gambale C, Piaggi P, Viola D, Giani C, Agate L, et al. Postoperative Thyroglobulin and Neck Ultrasound in the Risk Restratification and Decision to Perform 131I Ablation. J Clin Endocrinol Metab. 2017 Mar; 102(3):893-902.

19 American Thyroid Association (ATA) Guidelines Taskforce on Thyroid Nodules and Differentiated Thyroid Cancer, Cooper DS, Doherty GM, Haugen BR, Kloos RT, Lee SL, Mandel SJ, Mazzaferri EL, McIver B, Pacini F, Schlumberger M, Sherman SI, Steward DL, Tuttle RM: Revised American Thyroid Association management guidelines for patients with thyroid nodules and differentiated thyroid cancer. Thyroid. 2009 Nov;19(11):1167214.
20 Tsuda K, Namba H, Nomura T, Yokoyama N, Yamashita S, Izumi M, et al. Automated measurement of urinary iodine with use of ultraviolet irradiation. Clin Chem. 1995 Apr;41(4): 581-5.

21 Van Nostrand D. Selected Controversies of Radioiodine Imaging and Therapy in Differentiated Thyroid Cancer. Endocrinol Metab Clin North Am. 2017 Sep;46(3):783-93.

22 Pacini F, Molinaro E, Castagna MG, Agate L, Elisei R, Ceccarelli C, et al. Recombinant human thyrotropin-stimulated serum thyroglobulin combined with neck ultrasonography has the highest sensitivity in monitoring differentiated thyroid carcinoma. J Clin Endocrinol Metab. 2003 Aug;88(8):3668-73.

23 Durante C, Haddy N, Baudin E, Leboulleux S, Hartl D, Travagli JP, et al. Long-term outcome of 444 patients with distant metastases from papillary and follicular thyroid carcinoma: benefits and limits of radioiodine therapy. J Clin Endocrinol Metab. 2006 Aug;91(8): 2892-9. 